\title{
Arterial pulse waves measured with EMFi and PPG sensors and comparison of the pulse waveform spectral and decomposition analysis in healthy young and elderly subjects
}

\author{
M. Huotari ${ }^{1}$, A. Vehkaoja ${ }^{2}$, K. Määttä ${ }^{1}$, J. Kostamovaara ${ }^{1}$ \\ \& J. Röning ${ }^{1}$ \\ ${ }^{1}$ Oulu University, Oulu, Finland \\ ${ }^{2}$ Tampere University of Technology, Tampere, Finland
}

\begin{abstract}
The purpose of this study is to show the time domain and frequency domain analysis of signals recorded with electromechanical film (EMFi) and photoplethysmographic (PPG) sensors in arterial elasticity estimation via pulse wave decomposition and spectral components obtained from left forefinger, left wrist, and left second toe arteries. ECG and pulse waves from the subjects were recorded firstly from 20 voluntaries by PPG sensors and from 7 persons (3060 y) by parallel EMFi and PPG sensors in supine position. Decomposition of the pulse waves produces five components: percussion wave, tidal wave, dicrotic wave, and 1st and 2nd presystolic waves. Pulse wave decomposition parameters between EMFi and PPG are compared to find out information on a person's arterial elasticity. Results show that elastic information in the form of pulse wave decomposition from PPG waves is obtainable and shows clear shortening between percussion wave and tidal wave time with age. The spectral information obtained with frequency domain analysis could also be valuable in assessing the arterial elasticity.

Keywords: electromechanical film (EMFi), photoplethymography (PPG), pulse wave decomposition, arterial stiffness.
\end{abstract}




\section{Introduction}

Pulse waveform measurements by photoplethysmographic (PPG) and electromechanical film (EMFi) sensors are an effective method for arterial stiffness screening. A single pulse waveform comprises at least five components, namely, the percussion wave $f_{1}(t)$, the tidal wave $f_{2}(t)$, the dicrotic wave $f_{3}(t)$, the 1 st, and 2nd presystolic wave $\mathrm{f}_{4}(\mathrm{t})$ and $\mathrm{f}_{5}(\mathrm{t})$ as a whole. The last four are actually reflected waves as follows. After the left ventricular contraction the aorta valve opens and it is open as long time as the aorta pressure is lower than the ventricular pressure causing the percussion wave. During this time the aorta wall stores the elastic energy into its wall. To be exact the aorta valve closes when the aortic pressure gradient becomes negative during the last phase of ejection and the aortic wall will generate a tidal wave [1]. Also this negative pressure gradient will causethat blood flow decelerates. The percussion and the tidal wave travel along the arterial tree hitting to the bifurcation point where the arteries continue to the lower limbs. This hitting causes the dicrotic wave. All these waves can be measured from the fingertip and wrist by the PPG and EMFi method, respectively. The timing and amplitude of the reflected waves alter because of arterial stiffness changes. The wave pulse decomposition is used to estimate each wave component.

It is known that change of arterial elasticity is one of the early markers of accelerated arterial aging and can correlate with coronary risk factors. Especially arterial elasticity reflects the arterial and aortic expanding during left ventricular contraction. Arterial elasticity can be measured indirectly provided the measurement method is relevant and accurate enough. This biophysical function and structure of the arteries have been measured by PPG and EMFi sensors. For the measurement data we applied pulse wave decomposition (PWD), which reflects the elasticity of the aorta and its peripheral arteries. The combined PPG \&EMFi measurements can establish aortic and arterial elasticity based on PWD of the both signals during a heart cycle. So we do not need distance measurement required for pulse wave velocity estimation, which can be rather inaccurate in the case of the arterial tree [2]. The EMFi and PPG technologies require a few electromechanical or opto-electronic components: aEMFi film sensor connected to a high impedance amplifier, and a light source to illuminate the tissue, for instance finger tip, and a photodetector to measure the small variations in light absorbance amplified also by a high impedance amplifier. The recognized waveform feature is the peripheral pulse decomposed into five components. Despite its simplicity the origins of the different components of the PPG or EMFi signal are not fully understood [3]. We believe that these parallel pulse waves can provide valuable information about the circulatory system.

During a person's lifetime, as part of the ageing process or as a consequence of hypertension, atherosclerosis, hypercholesterolaemia, and other pathological processes make the aorta stiffen. In the elderly and in the stiffened arteries, the backward and forward wave merge together causing increasein pulse wave velocity (PWV). The arterial waves reflected from the periphery of the arterial tree, return earlier merging with the systolic part of the incident wave causing 
augmentation of the workload of the heart. Favourable softness between coupling of the left ventricle and the arterial tree is thus progressively lost. This loss can be greatest in the aorta, and least in the upper limbs. The wave reflection of the pulse wave due to increase in PWV also increases with age, but can be largely prevented by physical activity and proper diet. The amplitude spectrum of the ECG, EMFi, and PPG are changing by so-called integral pulse frequency modulation (IPFM) [4]. This modulation is caused because of the autonomic control mechanisms of cardiac functions which are involved in short-term fluctuations in the time interval between the consecutive heart cycles. The IPFM reflects cardiac function which is also detected in the periphery of the arterial tree. Healthy modulation in coupling of the left ventricle and the arterial tree is, however, progressively lost.

In our study we measure EMFi, two PPGs, and ECG signals, and we apply Fast Fourier Transform on these signals in addition to decomposition of the EMFi and PPG pulse waves.

\section{Methodology}

In this study, the PPG sensors are based on near infrared LEDs and photodetectors, EMFi sensors are based on plastic EMFi films, and ECG sensors are standard electrodes. Changes in light absorption, in the pressure, or in electrical potentials are acting on each sensor generating a measurable voltage. The EMFi sensor acts as a sensitive pressure sensor and the PPG sensor as a sensitive light absorbance sensor. Signals from ECG, PPG from 20 voluntaries and parallel PPG and EMFi sensors from 7 voluntaries were recorded with the PC healthy persons (20-60 y) using a data acquisition card and HP 356570A digital signal analyser with the sampling frequency of $500 \mathrm{~Hz}$. ECG signal was used as reference in detecting features from the PPG and EMFi related signals. Pulse wave series from EMFi, PPG finger and toe were processed by Origin software.

\section{Results and discussions}

Analysis in time domain is done for the pulse waves shown in Figure 1. Each pulse wave component from EMFi and PPG finger were processed by the software. Decomposed pulse waves from the left wrist (EMFi). Figure 2 shows actual pulse waves decomposed with their residual and confidence intervals, respectively.

In Figure 3, the integrals of residual plots presented in Figure 2 are shown for the selected variables (EMFi, PPG1) as a function of time. The residual sum of squares is then integrated producing residual integrals which represent the integral between the logarithmic function and the measured data points. The integral of the residual of the EMFi pulse wave is in the limit of $0.2 \%$, whereas finger PPG is about $0.7 \%$. The integrals of the both curves rise strongly during the systole and they have almost constant slope during the rest of the pulse wave duration. 

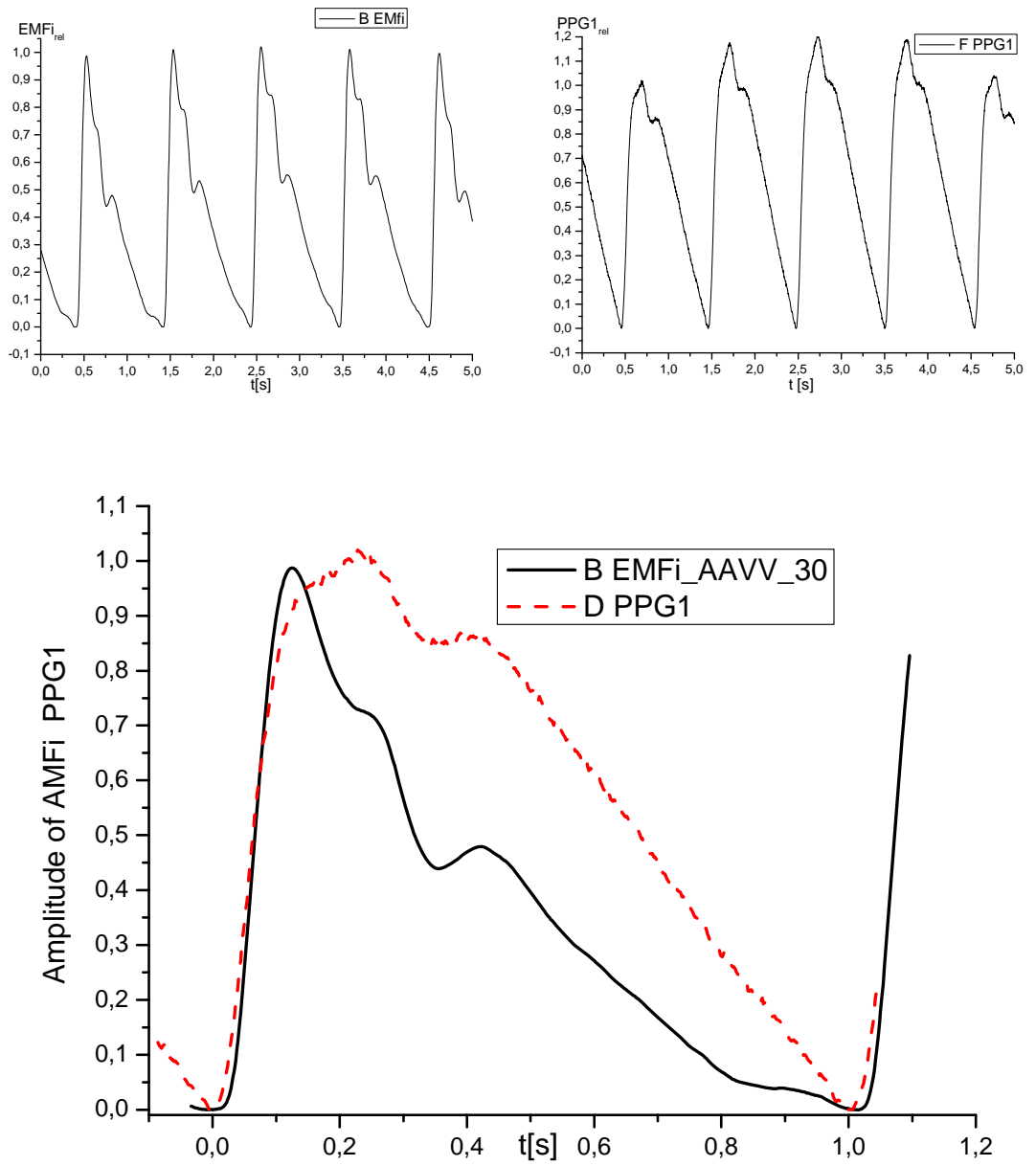

Figure 1: (Upper curves) An EMFi (left), and a PPG1 (right) pulse wave series (lower curves) a single pulse wave: EMFi (left wrist, black), PPG1 (left forefinger, red) (male 30).

Both the EMFi and PPG1 pulse wave show distinct and precise time relation when we look at the peak values of each component. The PPG signals contain personal variability which is repeated more often than in the EMFi pulse waves. However, we do not know exactly the cause and origin of this variability. A comparison of the PPG1 pulse waves shows that the tidal wave comes closer to the percussion wave peak value when person's age increases (Figure 2b) and 4b)). Also the comparison of the EMFi's and PPG1's amplitude spectra shows 

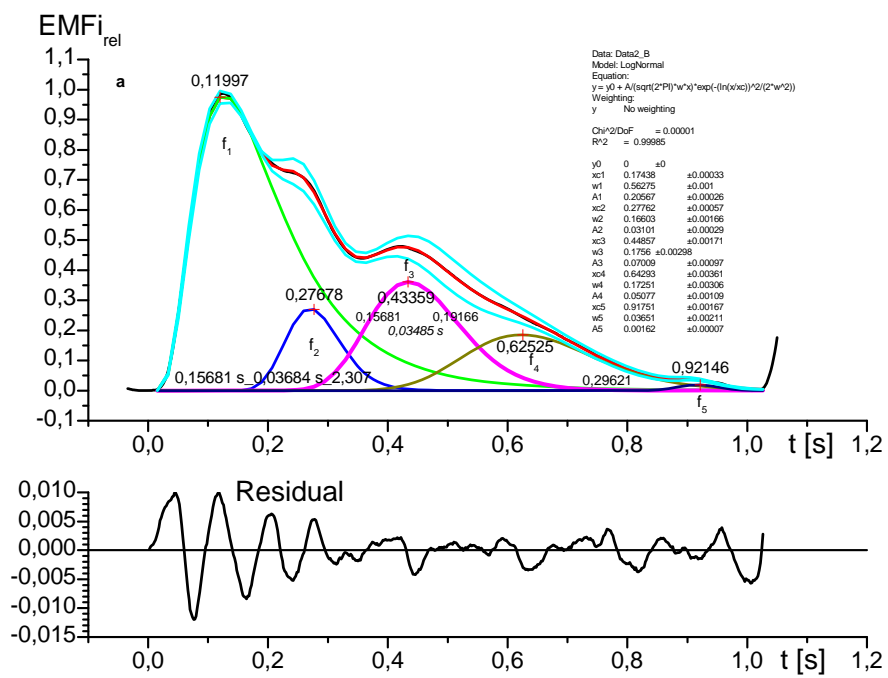

a)
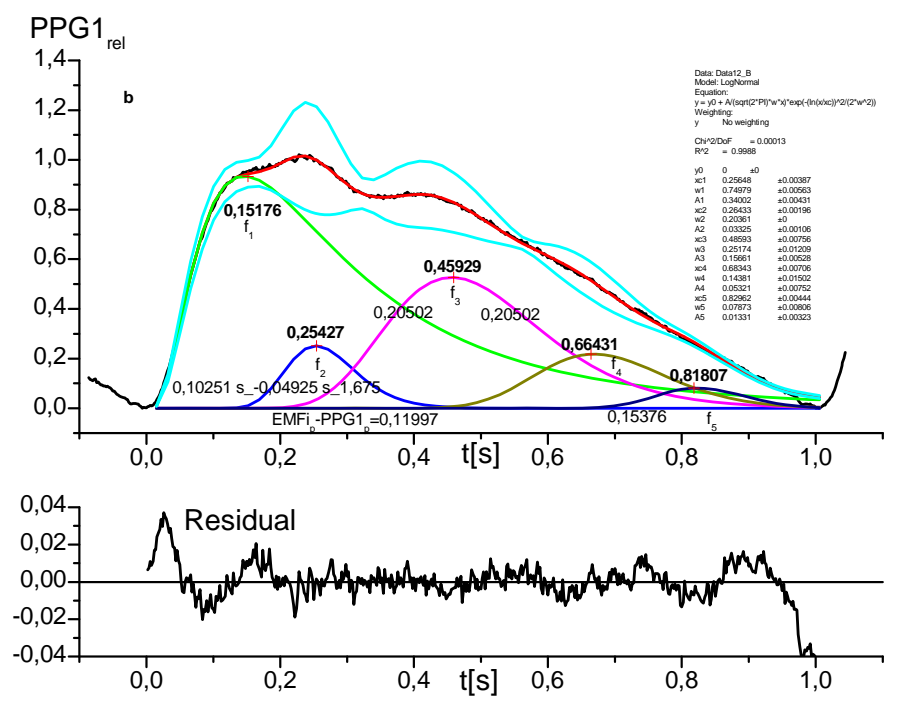

b)

Figure 2: a) A single EMFi pulse wave (black, measured) which is here decomposed into components: percussion wave (green, $\mathrm{f}_{1}$ ), tidal wave (blue, $\mathrm{f}_{2}$ ), dicrotic wave (violet, $\mathrm{f}_{3}$ ), 1st presystolic wave (dark yellow, $\mathrm{f}_{4}$ ), and 2 nd presystolic wave (navy, $\mathrm{f}_{5}$ ). The confidence interval (99\%) is cyan. Below the residual curve (black). b) A single PPG1 (finger) pulse wave decomposed (male 30). 


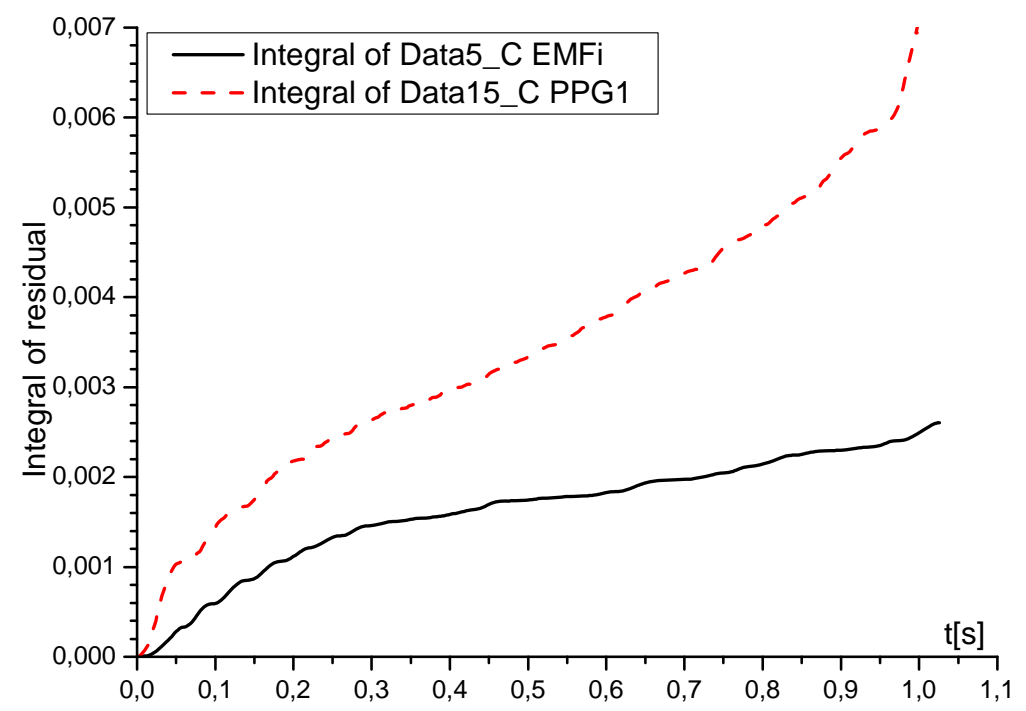

Figure 3: The integrals of the residual of the EMFi pulse wave (black) and the PPG1 (finger) pulse wave (red, dash) (male 30).

that the modulation frequency disappears or comes close to the carrier frequency as the person's age increases (Figures 5, 6, and 7). This study shows also that both the EMFi and PPG pulse waveforms can be decomposed to their component waves, namely, percussion wave, tidal wave, dicrotic wave, and the 1st and 2nd presystolic waves. Also the amplitude spectra of the respective pulse waves contain at least five or six components. The elastic information is obtainable from both EMFi and PPG pulse signals both in time and frequency domain because of the components time interval changes or the spectral changes clearly inspected. However, the information obtained from time domain (decomposition into logarithmic normal function) differs from that obtained from frequency domain because the latter contains many pulse waves which are IPFM modulated and they frequency components overlap. This information from the pulse wave propagation analysis can't be obtained. In scientific publications both the EMFi and PPG measurement method should be conclusively described when analyzing arterial pulse wave signals. 

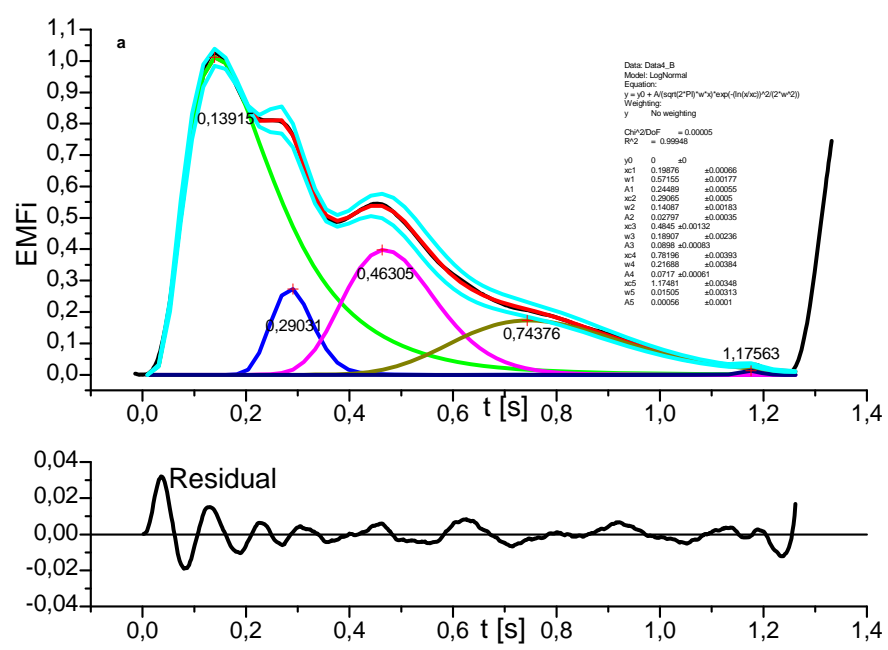

a)
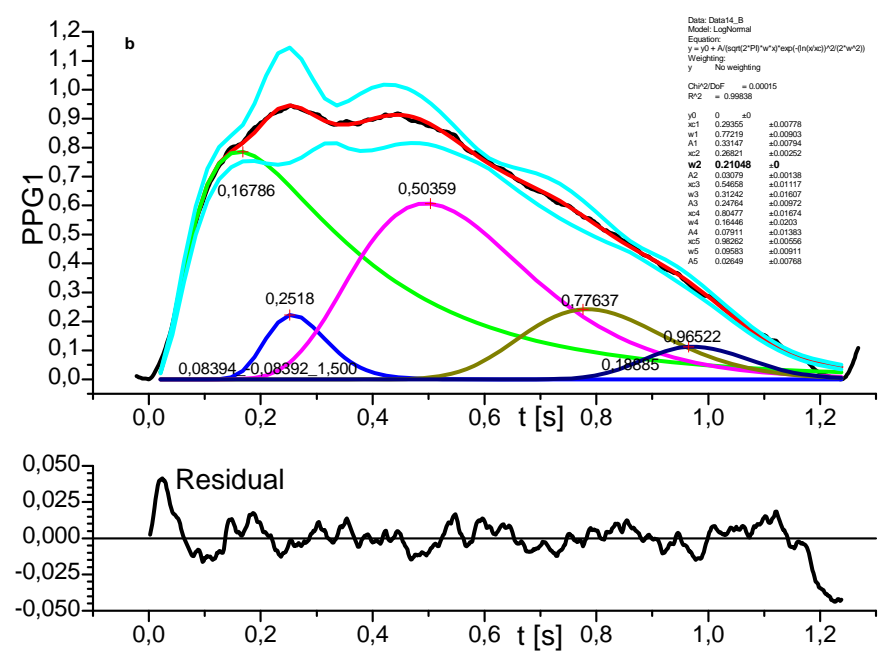

b)

Figure 4: a) A single EMFi pulse wave (black, measured) which is here decomposed into components: percussion wave (green), tidal wave (blue), dicrotic wave (violet), 1st presystolic wave (dark yellow), and 2nd presystolic wave (navy). The confidence interval (99\%) is cyan. Below the residual curve. b) A single PPG1 (finger) pulse wave decomposed (male 60). 

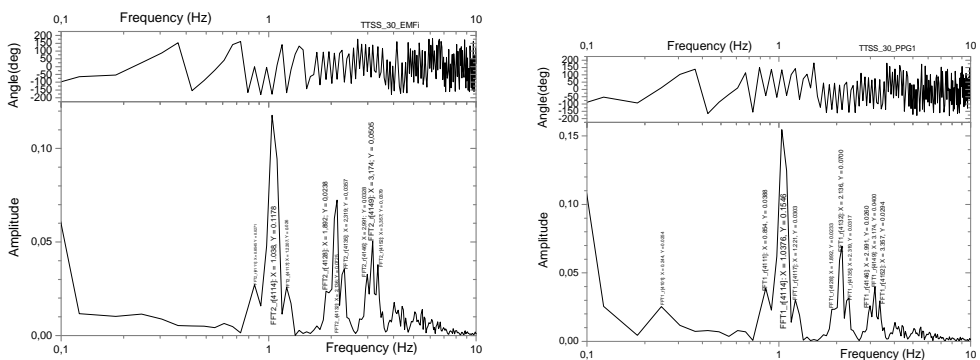

Figure 5: The pulse wave of EMFi's amplitude spectrum (left), and of PPG1's amplitude spectrum (right) with the IPFM parameter values for the three first components for 20 s sample record. In the PPG1's amplitude spectrum contains the breath rate frequency value (male 30).
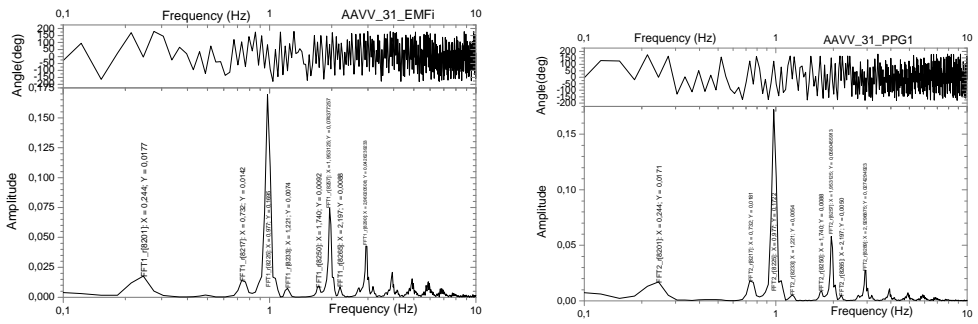

Figure 6: The pulse wave signal's amplitude spectrum (EMFi, (left), PPG1 (right)) with the IPFM parameter values for the three first components. The PPG1 and EMFi amplitude spectrum contain also the breath rate frequency value for $20 \mathrm{~s}$ signal record as a sample length (male 31).
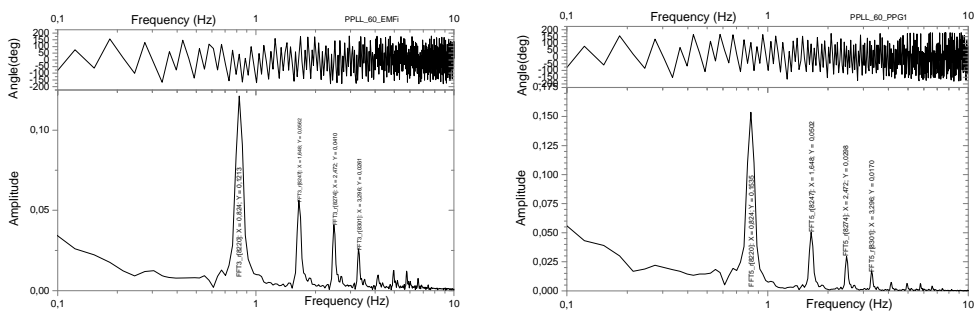

Figure 7: The EMFi pulse wave signal's amplitude spectrum (left), and of PPG1's amplitude spectrum (right). In the PPG1's and EMFi's amplitude spectrum contain also the breath rate frequency value (male 60). 


\section{References}

[1] Sugawara M., et al. (1977) Aortic blood momentum - the more the better for the ejecting heart in vivo? Cardiovascular Research, 33, 433-446.

[2] Alametsä J., Palomäki A. (2012) Comparison of local pulse wave velocity values acquired with EMFi sensor, Finnish Journal of eHealth and eWelfare 4, 89-98.

[3] Allen J. (2007) Photoplethysmography and its application in clinical physiological measurement, Physiological Measurement 28, R1-R39.

[4] Middleton P. M. et al. (2008) Spectral analysis of finger photoplethysmograpic waveform variability in a model of mild to moderate haemorrhage, Journal of Clinical Monitoring and Computing 22, 343-353. 